\title{
Maladaptive Sexual Behavior Following Concurrent Methamphetamine and Sexual Experience in Male Rats is Associated with Altered Neural Activity in Frontal Cortex
}

\author{
Lindsey B Kuiper ${ }^{1,2,5}$, Karla S Frohmader ${ }^{3,4,5}$ and Lique M Coolen ${ }^{* 1,3,4}$ \\ 'Department of Neurobiology and Anatomical Sciences, University of Mississippi Medical Center, Jackson, MS, USA; '² Graduate Program in \\ Neuroscience, University of Mississippi Medical Center, Jackson, MS, USA; ${ }^{3}$ Department of Anatomy \& Cell Biology, The University of Western \\ Ontario, London, Ontario, Canada; ${ }^{4}$ Department of Molecular \& Integrative Physiology, University of Michigan, Ann Arbor, MI, USA
}

\begin{abstract}
The use of psychostimulants is often associated with hypersexuality, and psychostimulant users have identified the effects of drug on sexual behavior as a reason for further use. It was previously demonstrated in male rats that methamphetamine (Meth), when administered concurrently with sexual behavior results in impairment of inhibition of sexual behavior in a conditioned sex aversion (CSA) paradigm where mating is paired with illness. This is indicative of maladaptive sex behavior following Meth and sex experience. The present study examined the neural pathways activated during inhibition of sexual behavior in male rats and the effects of concurrent Meth and sexual behavior on neural activity, using ERK phosphorylation (pERK). First, exposure to conditioned aversive stimuli in males trained to inhibit sexual behavior in the CSA paradigm increased pERK expression in medial prefrontal (mPFC), orbitofrontal cortex (OFC) and areas in striatum and amygdala. Second, effects of concurrent Meth and sex experience were tested in males that were exposed to four daily sessions of concurrent Meth $(\mathrm{I} \mathrm{mg} / \mathrm{kg})$ or saline and mating and subsequently exposed to CSA one week after last treatment. Meth and mating-treated males showed significant impairment of inhibition of mating, higher pERK expression under baseline conditions, and disrupted pERK induction by exposure to the conditioned aversive stimuli in mPFC and OFC. These alterations of pERK occurred in CaMKII-expressing neurons, suggesting changes in efferent projections of these areas. Altogether, these data show that concurrent Meth and mating experience causes maladapative sexual behavior that is associated with alterations in neural activation in mPFC and OFC.

Neuropsychopharmacology (2017) 42, 20 I I-2020; doi:I0.I038/npp.20I7.I; published online 8 February 2017
\end{abstract}

\section{INTRODUCTION}

There is increased awareness that drug addiction may share 'brain vulnerabilities' with the compulsive pursuit of nondrug rewards, such as gambling, sex, and food (Kraus et al, 2016). Abused drugs, including cocaine (Johnson and Bruner, 2012), methamphetamine (Meth) (Zapata et al, 2008), heroin (Mitchell et al, 2012), and alcohol (Mastroleo et al, 2015) are highly co-morbid with hypersexuality (Frohmader et al, 2010b). Drug users report heightened sexual desire, arousal, and pleasure (Green and Halkitis, 2006), loss of inhibitory control of sex behavior and increased risk taking behavior (Green and Halkitis, 2006; Semple et al, 2004). These effects are reported in men and women, heterosexuals and homosexuals (Cartier et al, 2008;

*Correspondence: Dr LM Coolen, Department of Physiology and Biophysics, University of Mississippi Medical Center, 2500N State Street, Jackson, MS 39216, USA, Tel: +60I 815 876I,

E-mail: Icoolen@umc.edu

${ }^{5}$ The authors contributed equally to this study.

Received I 3 September 20 I6; revised I 5 December 20 I 6; accepted 26 December 2016; accepted article preview online 4 January 2017
Johnson et al, 2015; Mausbach et al, 2009; Semple et al, 2004), and can serve as a primary motivation for drug use (Lyons et al, 2010). Thus, a better understanding of the neural basis for the association between drug use and hypersexuality may be beneficial for designing treatment options for both drug and sex addiction.

Our laboratory has shown that Meth treatment in male rats causes maladaptive sex behavior (Frohmader et al, 2010a, 2011), utilizing a conditioned sex aversion (CSA) paradigm (Davis et al, 2010) in which male rats learn to associate mating with subsequent visceral illness induced by lithium chloride ( $\mathrm{LiCl}$ ) (Peters, 1983). Following repeated pairings of mating and the illness, animals ceased to engage in copulatory behaviors (Davis et al, 2010; Frohmader et al, 2010a, 2011). However, males that received repeated experience with Meth exposure and mating behavior (ie, concurrent Meth and sex experience), continued to engage in sexual behavior in this CSA paradigm despite the learned negative consequences and weeks of drug abstinence before testing in the CSA paradigm (Frohmader et al, 2010c, 2011). This attenuating effect of Meth and sex experience was dependent on the concurrent experience of Meth and sex 
(Frohmader et al, 2011). These findings provide an experimental paradigm to further investigate the neural mechanisms by which concurrent Meth and mating experience causes maladaptive sexual behavior or hypersexuality.

The neural substrates of inhibition of sexual behavior are not understood. Therefore, the current study first investigated the neural circuits activated by cues associated with CSA using phosphorylation of MAP kinase, or extracellularregulated protein kinase ( $\mathrm{pERK}$ ), as a marker for neural activation in multiple brain regions known to be involved in sexual and drug reward behavior (Frohmader et al, 2010b, c), and aversion learning (Longoni et al, 2011). pERK is a useful marker for neural activation by conditioned cues and its expression is critical for learning and memory during different task including conditioned taste aversion (Berman et al, 1998), fear conditioning (Schafe et al, 2000), foodreward conditioning (Ribeiro et al, 2005), and sexual behavior (Pitchers et al, 2014). Moreover, the expression of pERK is involved in the mechanisms of drug action and synaptic plasticity (Giorgi et al, 2015; Valjent et al, 2006b).

Second, the hypothesis was tested that concurrent Meth and mating experience causes alterations in the neural circuits involved in inhibition of sexual behavior, by determining if concurrent Meth and sex experience disrupted conditioned aversive cue-induced pERK expression. Finally, to identify the particular neuronal projections that demonstrate disruption of pERK activation, cortical efferents, ie, pyramidal neurons, were identified by CaMKII expression (Fanous et al, 2012) and contrasted with cortical interneurons, identified by GAD expression (Fanous et al, 2012). Altogether, these approaches were used to test the hypothesis that concurrent Meth and mating experience disrupts neural circuits involved in inhibitory control of sexual behavior.

\section{MATERIALS AND METHODS}

\section{Subjects}

Adult male Sprague-Dawley rats (Charles River, Montreal, QC, Canada (experiments 1 and 2), Wilmington, MA (experiment 2 and Supplementary Study 3; 210-250 g)) were housed in pairs in standard Plexiglas cages on a $12 / 12 \mathrm{~h}$ reversed light/dark cycle. Food and water were available ad libitum. Female rats (Charles River, 201-225 g) for sexual partners were bilaterally ovariectomized under deep anesthesia ( $87 \mathrm{mg} / \mathrm{kg}$ ketamine and $13 \mathrm{mg} / \mathrm{kg}$ xylazine) and received a subcutaneous implant (Dow Corning, Midland, MI) containing 5\% estradiol benzoate (Sigma-Aldrich, St Louis, MO) and 95\% cholesterol (Sigma-Aldrich). Sexual receptivity was induced by a subcutaneous (s.c.) injection of $500 \mu \mathrm{g}$ progesterone (Sigma-Aldrich) in $0.1 \mathrm{ml}$ sesame oil (SigmaAldrich) $4 \mathrm{~h}$ before testing. All experimental procedures were conducted during dark phase and approved by the Institutional Animal Care and Use Committees at the University of Western Ontario, the University of Michigan, and the University of Mississippi Medical Center and conform to the guidelines outlined by the Canadian Council on Animal Care and the United States National Institutes of Health.

\section{Sexual Experience}

In experiment 1 , male rats $(n=11)$ gained sexual experience during 4 twice weekly mating sessions before the experiment. During each mating session, males were allowed to mate with a sexually experienced and hormone-treated receptive female, in separate test cages (mating arenas; $60 \times 45 \times 50 \mathrm{~cm}^{3}$ ) containing clean bedding, until the display of one ejaculation or for one hour, whichever occurred first. During each mating session, all standard parameters for sexual performance were recorded, including: mount latency (ML; time from introduction of the female until the first mount), intromission latency (IL; time from introduction of the female until the first mount with vaginal penetration), ejaculation latency (EL; time from the first intromission to ejaculation), post ejaculation interval (PEI; time from ejaculation to first subsequent intromission), number of mounts (M), and number of intromissions (IM) (Frohmader et al, 2011). All animals reached criteria of experience: three or more ejaculations during the four sessions (Supplementary Table 1).

\section{Concurrent Meth and Sex Experience}

In experiment 2, male rats received concurrent experience with Meth or saline exposure and sexual behavior. Thirty minutes before mating, males received either Meth $(1 \mathrm{mg} / \mathrm{kg}$ s.c.; Meth+Sex; $n=27)$ or saline (Sal+Sex; $n=26)$ and were subsequently allowed to mate to 1 ejaculation or for $1 \mathrm{~h}$, whichever occurred first, during 4 daily mating sessions on consecutive days. Meth treatment during these four sessions delayed initiation of mating (increased mount and intromission latencies; Supplementary Table 2), but did not affect measures of performance (ejaculation latencies; Supplementary Table 2), in agreement with our previous studies (Frohmader et al, 2010a). Moreover, groups did not differ in any measure of sexual behavior on the first day of CSA, confirming that the two groups were comparable at the onset of the CSA paradigm (Supplementary Table 2).

\section{CSA}

One (experiment 1) or two (experiment 2) weeks following the last mating session, animals were subjected to a CSA paradigm using $\mathrm{LiCl}$ as described in our previous papers (Frohmader et al, 2010a, 2011). Briefly, animals were exposed to seven (experiment 1) or eight (experiment 2) consecutive conditioning trials, each consisting of 2 days. On the first day, males were placed in the mating arena for a 10 min habituation period after which a receptive female was introduced, scented by swabbing almond oil on the neck and base of the tail to strengthen conditioning (Agmo, 2002). Males mated for $30 \mathrm{~min}$ or until one ejaculation. If intromission did not occur within the first $15 \mathrm{~min}$, mating was terminated. One minute following ejaculation or trial termination all males received a $127.2 \mathrm{mg} / \mathrm{kg}$ intraperitoneal (i.p.) injection of $\mathrm{LiCl}$ administered at a volume of $10 \mathrm{ml} / \mathrm{kg}$. $\mathrm{LiCl}$ was administered regardless of whether mating occurred. The second day of each trail, males received saline injections, but were not exposed to scented females or allowed to mate. Our previous papers have shown significant inhibition of initiation of sexual behavior in male rats 
following three or more conditioning trials when tested 1-2 weeks after gaining sexual experience during daily or twice weekly sessions (Davis et al, 2010; Frohmader et al, 2010a, 2011).

\section{Final Test}

Following the final conditioning trial, males were exposed to either the sex aversion conditioned cue, ie, the scented female and the mating cage environment, and perfused $10 \mathrm{~min}$ later to examine pERK expression, or removed from their home cage before brain collection to serve as controls. The expression of pERK is highly dynamic and occurs 5-20 min after neural activation (Frohmader et al, 2010c). Groups in experiment 1 included: exposed to Female $(n=6)$ or Home Cage control $(n=6)$. Groups in experiment two included: Sal+Sex Home Cage $(n=12)$, Sal+Sex Female $(n=14)$, Meth+Sex Home Cage $(n=10)$, and Meth+Sex Female $(n=17)$. Mating behavior was not displayed by any of the males exposed to the scented female during this final test. Experiment 2 was conducted in two separate replicate cohorts of animals, each containing the same experimental groups and using identical methods. This was due to relocation of the laboratory (half of each group was tested at the University of Western Ontario and half at the University of Michigan). The two replicates of the experiment did not statistically differ in any of the measured parameters (outlined below) and were combined for final analysis.

\section{Tissue Preparation}

Animals were anesthetized with pentobarbital $(270 \mathrm{mg} / \mathrm{kg}$; i.p., Bimeda-MTC, Animal Health, Cambridge, ON, Canada; Vortech, Dearborn, MI, USA) and perfused transcardially with $5 \mathrm{ml}$ of saline followed by $500 \mathrm{ml} 4 \%$ paraformaldehyde (Electron Microscopy Sciences, Hatfield, PA) in $0.1 \mathrm{M}$ phosphate buffer (PB). All perfusions were performed within 4 min of pentobarbital injection to avoid any effects of pentobarbital or injection stress on pERK immunoreactivity (Takamura et al, 2008). Brains were removed and post-fixed for $1 \mathrm{~h}$ at room temperature in the same fixative, then immersed in 20\% sucrose (Fisher Scientific, Fair Lawn, NJ) and $0.01 \%$ Sodium Azide (Sigma-Aldrich) in $0.1 \mathrm{M} \mathrm{PB}$ and stored at $4{ }^{\circ} \mathrm{C}$. Coronal sections $(35 \mu \mathrm{m})$ were cut on a freezing microtome (H400R, Micron, Germany), collected in four parallel series in cryoprotectant solution (30\% sucrose and $30 \%$ ethylene glycol in $0.1 \mathrm{M} \mathrm{PB}$ ) and stored at $-20^{\circ} \mathrm{C}$ until further processing.

\section{Immunohistochemistry}

All incubations were performed at room temperature with gentle agitation. Free floating sections were washed extensively with phosphate-buffered saline $(0.1 \mathrm{M} \mathrm{PB}$ containing $0.9 \%$ sodium chloride; PBS) between incubations. Sections were incubated in $1 \% \mathrm{H}_{2} \mathrm{O}_{2}$ for $10 \mathrm{~min}$, then blocked in incubation solution (PBS containing $0.1 \%$ bovine serum albumin and $0.4 \%$ Triton X-100) for $1 \mathrm{~h}$.

pERK. One parallel series of sections from each animal was processed to visualize pERK using immunoperoxidase technique. Sections from all experimental groups within each of the two experiments were immunoprocessed simultaneously. Sections were incubated overnight with a rabbit polyclonal antibody against p42 and p44 MAP Kinases ERK1 and ERK2 (pERK; 1:4000; Cell Signaling Technology, Danvers, MA, USA Cat \# 9101), followed by a $1 \mathrm{~h}$ incubation with biotinylated goat anti-rabbit IgG (1:500; $1 \mathrm{~h}$; Vector Laboratories, Burlingame, CA, USA) and avidin-horseradish peroxidase complex (ABC-Elite; 1:1000; Vector). Next, tissue was incubated for $10 \mathrm{~min}$ in a chromogen solution containing $0.02 \%$ 3,3'-diaminobenzidine tetrahydrochloride (DAB; Sigma-Aldrich) in $0.1 \mathrm{M} \mathrm{PB}$ containing $0.015 \%$ hydrogen peroxide, resulting in a reddish-brown reaction product. Sections were washed thoroughly in $0.1 \mathrm{M} \mathrm{PB}$, mounted onto Superfrost plus glass slides (Fisher, Pittsburgh, PA, USA) with $0.3 \%$ gelatin in $\mathrm{ddH}_{2} \mathrm{O}$. Following $\mathrm{DAB}$ staining, slides were dehydrated and coverslipped with dibutyl phthalate xylene (DPX; Sigma-Aldrich).

Dual fluorescence for pERK+CaMKII and pERK+GAD67. One parallel series of sections from experiment 2 containing mPFC and orbitofrontal cortex (OFC) were incubated overnight with either mouse anti-CaMKII (1:10 000; Cat\# MA1-048, Pierce Biotechnology, Rockford, IL) or mouse anti-GAD67 (1:200; Cat\#MAB5406, lot 2188494, Millipore, Temecula, CA) and Alexa Fluor 555-conjugated goat antimouse secondary (1:100; $30 \mathrm{~min}$; Cat\# A21422, Molecular Probes, Eugene, OR). Next, sections were incubated overnight with rabbit anti-pERK (1:4000; overnight; Cell Signaling, Cat\# 9101) and with biotinylated goat anti-rabbit for one hour (1:500; cat. \# BA-1000, Vector Laboratories, Burlingame, CA, USA), avidin-horseradish peroxidase complex (ABC-elite, 1:1000 in PBS; $1 \mathrm{~h}$; cat. \# PK-6100, Vector), biotinylated tyramine/tissue sample amplification (TSA; 1:250 in PBS containing $1 \mathrm{ul} / \mathrm{ml}$ of $3 \% \mathrm{H} 2 \mathrm{O} 2$ for $10 \mathrm{~min}$; NEL700/700A; PerkinElmer Life Sciences, Boston, MA, USA), and Dylight 488 conjugated streptavidin (1:100 in PBS, $30 \mathrm{~min}$; cat. \#21832, lot PF204220, Pierce Biotech., Rockford, IL, USA). Sections were mounted on plus charged slides (Thermo Fisher Scientific, Cat\# 12-550-20, Waltham, MA, USA) and coverslipped with an aqueous mounting medium (Gelvatol; (Harlow and Lane, 1988)) containing the anti-fading agent 1,4-diazabicyclo(2,2)octane (DABCO; $50 \mathrm{mg} / \mathrm{ml}$, Sigma-Aldrich) and stored in the dark at $4{ }^{\circ} \mathrm{C}$.

All primary antibodies have been extensively characterized in rat, omission of the primary antibodies prevented all immunoreactivity, and Western blot analysis of rat brain tissue revealed bands at the appropriate molecular weights for all antibodies (Fanous et al, 2012).

\section{Data Analysis}

SigmaPlot (Systat Software, San Jose, CA) was used for all statistical analyses.

Sexual behavior. Standard parameters for sexual performance (latencies to mount, intromission, ejaculation, and numbers of mounts and intromissions) were each compared between groups using a Fisher exact test. Data analysis of sexual behavior before CSA revealed no significant 
differences between groups in any of the parameters of sexual performance.

For the CSA paradigm, percentages of males that displayed one or more mounts, intromissions, or ejaculation during a conditioning trial were calculated and compared with conditioning trial 1 using a Fisher exact test at a significance level of 0.05 . In addition, and in experiment 2, differences between groups within each of the conditioning trials were compared using the Fisher exact test.

pERK cell counts. Single-labeled DAB-stained cells for pERK were counted in the nucleus accumbens (NAc) core and shell subregions, the caudate putamen $(\mathrm{CPu})$, basolateral amygdala (BLA), lateral amygdala (LA), central amygdala $(\mathrm{CeA})$, the OFC, the anterior cingulate area (ACA), prelimbic (PL), and infralimbic (IL) subregions of the medial prefrontal cortex (mPFC), and the ventral tegmental area (VTA) using standard areas of analysis using a camera lucida drawing tube attached to a Leica DMRD microscope (Leica Microsystems, Wetzlar, Germany). Standard areas of analysis were: $400 \times 600 \mu \mathrm{m}$ in the NAc core and shell subregions and LA, $800 \times 800 \mu \mathrm{m}$ in the $\mathrm{CPu}, 600 \times 600 \mu \mathrm{m}$ in the lateral OFC, $600 \times 800 \mu \mathrm{m}$ in the BLA, CeA, and each subregion of the $\mathrm{mPFC}$, and $800 \times 1200 \mu \mathrm{m}$ in the VTA (Supplementary Figure 1). Two sections were counted for each brain region. Cell numbers per $\mathrm{mm}^{2}$ were calculated, and the two counts were averaged per animal. Group averages were compared using a Student's $t$-test at significance level of 0.05 (experiment 1) or two way ANOVA (factors: Meth vs saline, and home cage control $v s$ female exposure; experiment 2) and Holm-Sidak pairwise post-hoc comparisons at a significance level of 0.05 (experiment 2).

pERK/CAMKII or $p E R K / G A D$. In fluorescent-stained sections from experiment 2, cells labeled for pERK, CaMKII or GAD67, or dual were counted in standard areas of analysis as described above in experiment 2 for immunoperoxidase-stained sections in ACA, IL, PL, lateral OFC and an additional counting area ventral within the lateral OFC $(600 \times 600 \mu \mathrm{m})$, using MicroBrightField Neurolucida software (Williston, Vermont, USA). The percentages of co-expression were calculated per section and averaged per animal. Group averages were compared using two way ANOVA (factors: Meth vs saline and home cage control vs female exposure) and Holm-Sidak pairwise post-hoc comparisons at a significance level of 0.05 .

\section{RESULTS}

\section{Neural Activation of the Limbic System Following CSA}

In experiment 1 , conditioning for sex aversion significantly inhibited sexual behavior (Table 1 and Figure 1a), confirming our previous findings (Davis et al, 2010; Frohmader et al, 2011). Specifically, decreased percentages of LiCl-paired males that displayed mounts, intromissions, or ejaculations were first evident on the fourth conditioning day and persisted through conditioning days 5-7 (Table 1).

Analysis of numbers of pERK cells in animals from experiment 1 revealed that exposure to the conditioned cue associated with sex aversion (ie, the scented female) significantly increased pERK expression in regions of the
Table I Percentages of Males $(n=\mid 1)$ in Experiment | Displaying One or More Mount, Intromission, or Ejaculation During Each of Seven Conditioning Sessions of the CSA Paradigm

\begin{tabular}{lccccccc}
\hline & CD1 & CD2 & CD3 & CD4 & CD5 & CD6 & CD7 \\
\hline \% Mounting & 100 & 100 & 88.9 & $66.7^{\mathrm{a}}$ & $66.7^{\mathrm{a}}$ & $27.8^{\mathrm{a}}$ & $61.1^{\mathrm{a}}$ \\
\% Intromitting & 100 & 94.4 & 88.9 & $66.7^{\mathrm{a}}$ & $66.7^{\mathrm{a}}$ & $27.8^{\mathrm{a}}$ & $61.1^{\mathrm{a}}$ \\
\% Ejaculating & 94.4 & 94.4 & 88.9 & $44.4^{\mathrm{a}}$ & $50^{\mathrm{a}}$ & $11.1^{\mathrm{a}}$ & $33.3^{\mathrm{a}}$
\end{tabular}

andicates significant difference from CDI (Fisher exact; CD: conditioning day): CD4: $M$ and I: $p=0.02, E ; p=0.003 ; C D 5: M$ and I: $p=0.02, E: p=0.007 ; C D 6:$ M, I, E: $p \leqslant 0.001$; and CD7: $M$ and I: $p=0.008, E ; p \leqslant 0.001$.

frontal cortex, striatum, and amygdala (Figure $1 \mathrm{~b}-\mathrm{d}$ and Figure 2). Specifically, exposure to the scented female increased $\mathrm{pERK}$ expression in the ACA, PL, and IL regions of the mPFC (Figure $1 b ; p=0.037, p=0.036, p=0.047$ ), OFC $(p=0.020)$, NAc shell $(p=0.045), \mathrm{CPu}(p=0.032)$, and CeA $(p=0.023)$ compared with home cage controls (Figure 1c and $d$ ). In contrast, induction of $\mathrm{pERK}$ immunoreactivity was not observed in the NAc core, in the lateral and basolateral amygdala, or in the ventral tegmental area (Figure $1 c$ and $\mathrm{d}$ ).

\section{Meth and Sex Pretreatment Attenuates Neural Activation Following CSA and Increases Baseline Neural Activation}

In experiment 2, concurrent Meth and sex experience attenuated CSA (Table 2 and Figure 3a) confirming our previous observations (Frohmader et al, 2010a, 2011). Significantly higher percentages of males pre-exposed to Meth and sex displayed mounts, intromissions, and ejaculations compared with males treated with saline and sex (Table 2). Group differences were first observed on the fifth conditioning day, and persisted through conditioning days 6 , 7, and 8. In addition, saline and sex-pretreated animals showed a significant inhibition on and after conditioning day 6, whereas Meth and sex-pretreated animals showed significant inhibition only during conditioning day 8 (Table 2).

Analysis of pERK in animals from experiment 2 showed that there were main effects of exposure to the conditioned aversive stimulus on pERK in the PL and IL subregions of the mPFC (Figure 3; $\mathrm{F}(1,49)=5.1$ and $5.2 ; p=0.028$ and $0.027)$, NAc core $(\mathrm{F}(1,47)=5.8 ; p=0.020)$ and shell $(\mathrm{F}(1,47)=4.5 ; p=0.039)$, CeA $(\mathrm{F}(1,49)=20.3 ; p<0.001)$, and $\mathrm{CPu}(\mathrm{F}(1,49)=12.3 ; p<0.001)$. In addition, there was a main effect of Meth pretreatment in all subregions of the $\mathrm{mPFC}$ (Figure 3b; $\mathrm{F}(1,49)=1.1-4.209 ; p=0.014-0.046$ ), and OFC $(\mathrm{F}(1,48)=7.7 ; p=0.008)$, and an interaction in OFC $(\mathrm{F}(1,48)=7.6 ; p=0.008)$.

Post-hoc analyses showed that in saline-pretreated males, exposure to the female increased pERK in ACA, $(p=0.040)$, PL $(p=0.010)$ and IL $(p=0.004)$, OFC $(p=0.005)$, CeA $(p<0.001)$, NAc core $(p=0.010)$ NAc shell $(p=0.041)$, and $\mathrm{CPu}(p=0.013)$ compared with saline-pretreated home cage controls (Figure $3 \mathrm{~b}$ and $\mathrm{c}$ ), confirming the findings of experiment 1. This pattern of activation of pERK by conditioned aversive cues was different in males that 
a

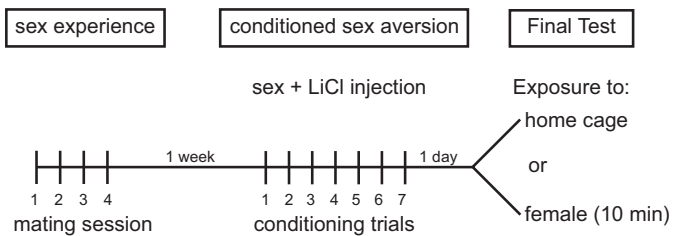

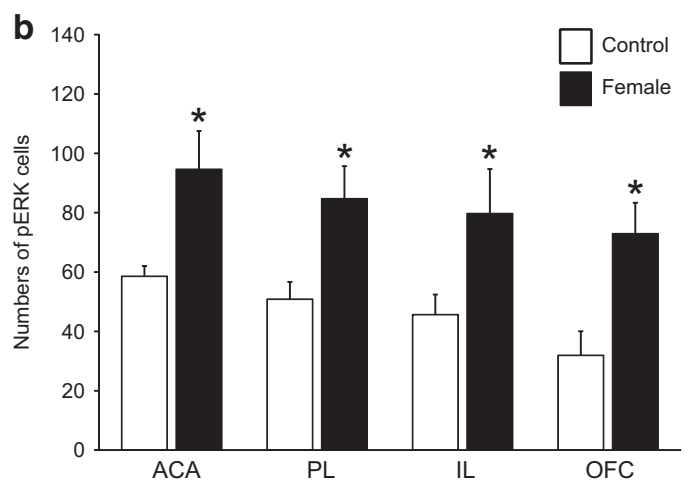

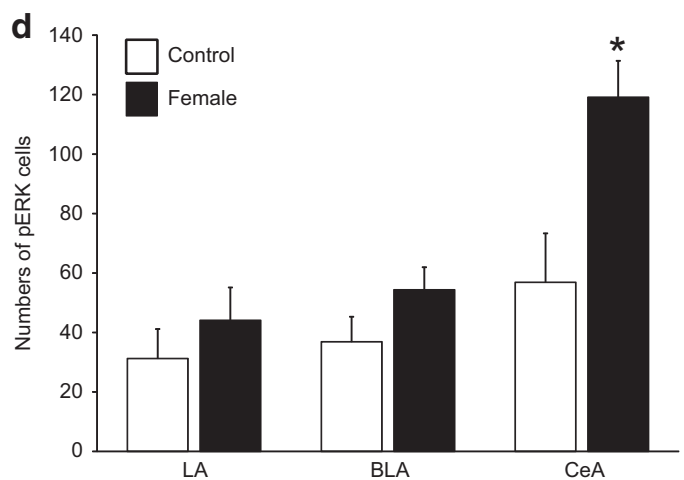

Figure I Conditioned aversive cue-induced phosphorylated MAP kinase (pERK) after conditioned sex aversion (CSA) (experiment I). (a) Experimental design and timeline of experiment I showing 4 days of sex experience, followed I week later by 7 conditioning trials of CSA, and the final exposure to home cage or female for pERK analysis. (b-d) Numbers of pERK-immunoreactive neurons per $\mathrm{mm}^{2}$ (mean \pm SEM) in control males removed from home cage (control, white bars, $n=5$ ) or males exposed to the conditioned aversive cues, ie females (female, black bars, $n=6$ ). Cell counts are shown for (b) subregions of the medial prefrontal cortex (mPFC) and orbitofrontal cortex (OFC) (ACA, anterior cingulate area; PL, prelimbic cortex; IL, infralimbic cortex); (c) striatum and VTA (NAc, nucleus accumbens Core and Shell; CPU, caudate putamen; VTA, ventral tegmental area), and (d) amygdala (LA, lateral amygdala; BLA, basolateral amygdala; CeA, central amygdala). *Significant difference from home cage control group.

received pretreatment of Meth and sex experience, as exposure to the aversive cues did not induce pERK in the NAc core or shell, mPFC subregions, or OFC compared with Meth-pretreated home cage controls. In contrast, cue exposure in Meth-pretreated males did induce pERK in $\mathrm{CPu}(p=0.011)$ and $\mathrm{CeA}(p=0.029)$ compared with Methtreated home cage controls (Figure $3 c$ ).

In addition, Meth pretreatment increased baseline (home cage) pERK expression in the $\operatorname{mPFC}(p=0.022, p=0.042$ and 0.021 ; ACA, PL, and IL) and OFC $(p<0.001)$ compared with saline-treated home cage controls (Figure $3 \mathrm{~b}$ ). This effect did not reach statistical significance in the NAc core and shell, $\mathrm{CPu}$ or $\mathrm{CeA}$ (Figure $3 \mathrm{c}$ ).

To rule out effects of exposure to the CSA paradigm on this increased baseline pERK expression, experiment 2 was replicated in a separate study in which males received concurrent or non-concurrent Meth and sex exposure without testing in the CSA paradigm (Supplementary Materials: experiment 3). In this separate study, concurrent Meth and sex pretreatment increased pERK expression under baseline conditions (Supplementary Figure 2), replicating the findings of experiment 2. Therefore, exposure to CSA paradigm was not the cause for the baseline increase. Moreover, this effect was not evident in males that received non-concurrent Meth and sex experience (Supplementary
Figure 2), indicating that concurrent Meth and sex experience is essential for this alteration in baseline activity and that the effect appears to be synergistic, rather than additive. Finally, supplementary analysis of pERK was conducted in experiment 2 in the portion of saline-treated animals that showed higher rates of initiation (ie, persistence) of mating behavior despite learned negative consequences (Supplementary Data, analysis experiment 2), confirming an increased pERK baseline expression in animals that show maladaptive sex behavior without Meth pretreatment (Supplementary Table 3).

\section{pERK Expression in CaMKII, But Not GAD65 Cells}

To next determine if pERK is expressed in mPFC and OFC pyramidal neurons or interneurons, analysis of dual fluorescent-stained sections for $p E R K$ and CaMKII (expressed in pyramidal cells (Fanous et al, 2012)) or GAD (expressed in interneurons (Fanous et al, 2012)) was performed. First, the findings above for pERK expression were confirmed using the fluorescent analysis (Figure 4a: Main effects of Meth: ACA: $\mathrm{F}(1,11)=5.8 ; p=0.034$, PL: $\mathrm{F}(1,10)=18.7 ; p=0.002$, IL: $\mathrm{F}(1,10)=8.6 ; p=0.015$, OFC: $\mathrm{F}(1,14)=10.8 ; p=0.005$; Main effect of female: OFC: $\mathrm{F}(1,14)=6.5, \quad p=0.023$; and interaction effects: ACA: $\mathrm{F}(1,11)=7.4, \quad p=0.020 ; \quad \mathrm{PL}: \mathrm{F}(1,10)=6.6, \quad p=0.028 ;$ IL: 

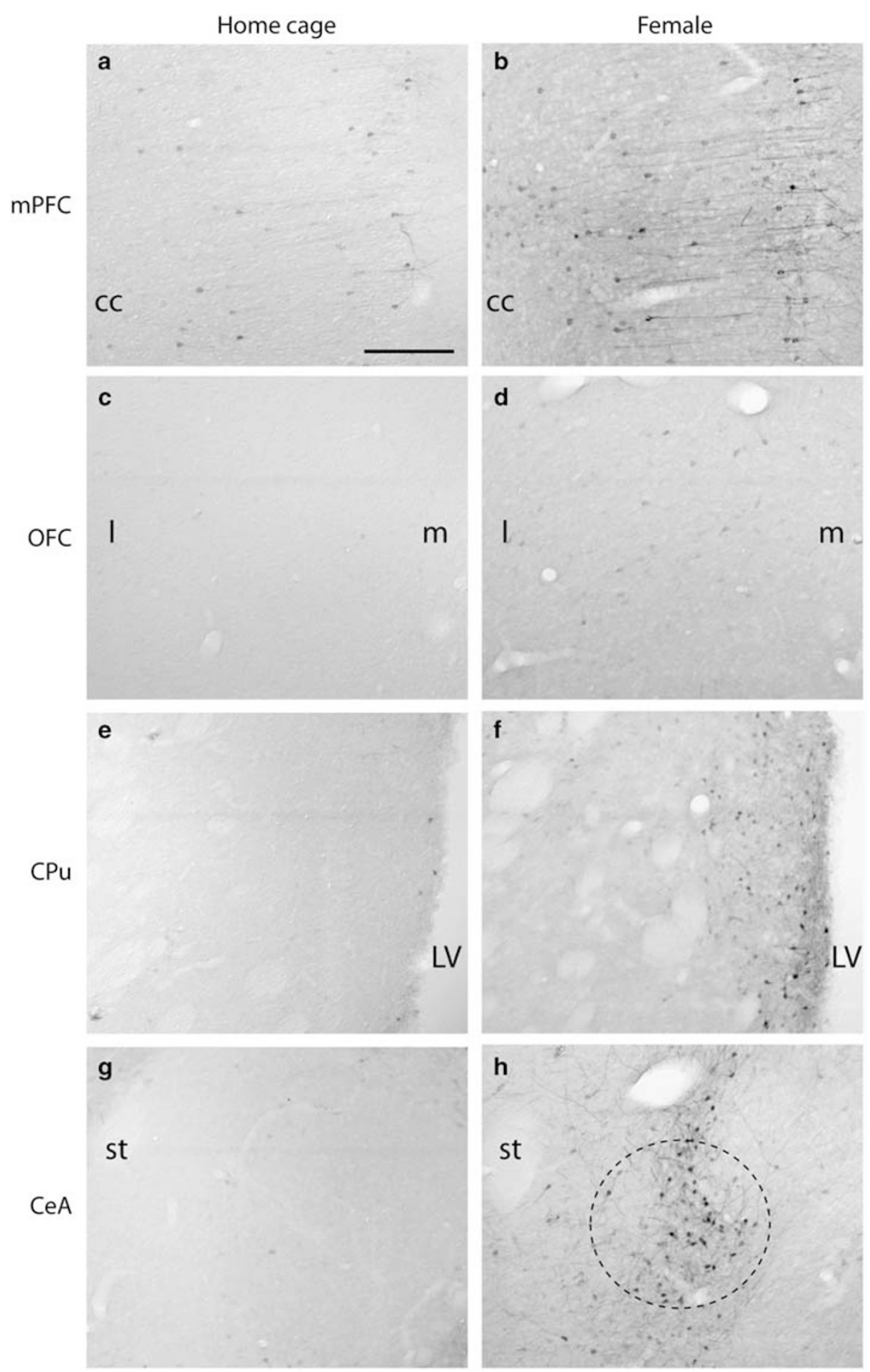

Figure 2 Representative images of pERK expression in home cage control males (a,c,e,g) and following exposure to the conditioned aversive cue, ie, the female (b,d,f,h) in experiment I. CeA, central amygdala; cc, corpus callosum; CPu, caudate putamen; LV, lateral ventricle; mPFC, medial prefrontal cortex; OFC, orbitofrontal cortex (I: lateral; m: medial); st, stria terminalis. Circle in h indicates location of CeA. Scale bar indicates $200 \mu \mathrm{m}$.

$\mathrm{F}(1,10)=8.6, p=0.015$; OFC: $\mathrm{F}(1,14)=16.5, p=0.001)$. Posthoc analyses showed that exposure to the female increased pERK in saline-pretreated control males compared with home cage controls (Figure 4a; ACA: $p=0.027$; PL: $p=0.033$; IL: $p=0.045$; OFC: $p=0.002$ ). Meth-pretreated males had increased pERK expression under baseline conditions
(ACA: $p=0.009, \quad$ PL: $p=0.001$, IL: $p=0.004$, OFC: $p=0.001$ compared with saline home cage controls) and exposure to the female did not induce further pERK expression (Figure 4a).

Moreover, analysis of dual labeling showed that mPFC pERK was expressed in CaMKII neurons in all groups in 
Table 2 Percentages of Males Displaying Mating Behavior During Sex Aversion Conditioning After Saline $(n=26)$ or Meth $(n=27)$ Pretreatment in Experiment 2

CDI CD2 CD3 CD4 CD5 CD6 CD7 CD8

\begin{tabular}{lllllllll}
\hline Saline & & & & & & & & \\
\% Mounting & 92.3 & 96.2 & 92.3 & 84.6 & 73.1 & $42.3^{\mathrm{a}}$ & $53.8^{\mathrm{a}}$ & $46.2^{\mathrm{a}}$ \\
\% Intromitting & 92.3 & 96.2 & 92.3 & 84.6 & 73.1 & $42.3^{\mathrm{a}}$ & $53.8^{\mathrm{a}}$ & $46.2^{\mathrm{a}}$ \\
\% Ejaculating & 84.6 & 80.8 & 92.3 & 76.9 & 61.5 & $34.6^{\mathrm{a}}$ & $46.2^{\mathrm{a}}$ & $34.6^{\mathrm{a}}$
\end{tabular}

Meth

$\begin{array}{lcccccccc}\text { \% Mounting } & 92.6 & 100 & 92.6 & 100 & 96.3^{\mathrm{b}} & 100^{\mathrm{b}} & 88.9^{\mathrm{b}} & 66.7^{\mathrm{a}} \\ \text { \% Intromitting } & 88.9 & 100 & 92.6 & 100 & 92.6 & 96.3^{\mathrm{b}} & 88.9^{\mathrm{b}} & 66.7 \\ \text { \% Ejaculating } & 81.5 & 85.2 & 92.6 & 92.6 & 66.7 & 81.5^{\mathrm{b}} & 81.5^{\mathrm{b}} & 55.6^{\mathrm{a}}\end{array}$

andicates significant difference from CDI within group (Fisher exact; CD: conditioning day): CD6 saline, $M$, I, and E: $p<0.00$ I; CD7 saline, $M: p=0.004$, I: $p=0.004, E: p=0.008 ;$ CD8 saline, M, I, and E: $p<0.00$ I; CD8 Meth, M:

$p=0.039, E: p=0.047$. Indicates significant difference from saline-pretreated males for each conditioning day (Fisher exact): CD5, M: $p=0.024 ; C D 6, M$, I, and E: $p<0.00$ I; CD7, $M$ and I: $p=0.006, E: p=0.010$.

both $\mathrm{mPFC}$ and OFC (Figure $4 \mathrm{~b}$ and $\mathrm{c}-\mathrm{h}$ ). Analysis of pERK expression in CaMKII neurons mirrored that of pERK alone (Figure 4b; Main effects of Meth: $\mathrm{F}(1: 10-14=5.3-18.1$, $p=0.002-0.045$; Main effects of female: $\mathrm{F}(1: 10-14)=8.7$ $12.3, p=0.005-0.028)$ ). Post-hoc analyses showed that in the ACA, PL, and IL subregions of the mPFC, pERK was increased in CaMKII cells by exposure to the female in saline and sex-pretreated males ( $p=0.012,0.007,0.019)$, but not in Meth and sex-pretreated males. Meth and sex-pretreated males had significantly higher pERK expression in CaMKII cells in the ACA, PL, and IL subregions of the MPFC under baseline conditions compared with saline and sex-treated males (Figure $4 \mathrm{~b} ; p=0.015,0.002,0.010$ ). In the OFC, pERK was increased in CaMKII cells by exposure to the female in saline and sex-pretreated males $(p=0.046)$, but not in Meth and sex-pretreated males. Finally, in all groups pERKimmunoreactive cells were observed that were not co-labeled for CaMKII: 33-50\% of pERK cells in mPFC subregions and $17-22 \%$ of pERK cells in OFC co-expressed CaMKII, regardless of home cage control or female exposure. However, single-labeled pERK cells did have the morphological appearance of pyramidal cells, suggesting that CaMKII staining failed to identify all pyramidal neurons (Figure $4 \mathrm{c}-\mathrm{e}$ and $\mathrm{f}-\mathrm{h}$ ). Finally, pERK cells did not co-express GAD $(0-0.3 \%)$ in any of the groups (Figure $4 \mathrm{i}-\mathrm{k})$.

\section{DISCUSSION}

These findings indicate that concurrent Meth and sex experience may cause maladaptive sexual behavior by increasing baseline neural activity in $\mathrm{MPFC}$ and $\mathrm{OFC}$, and by attenuating neural activation in $\mathrm{MPFC}$, OFC, and NAc induced by conditioned cues signaling aversive outcomes of sex behavior. These findings provide new insights into the neural circuits that are affected in drug-associated hypersexuality. Moreover, neural activity changes occurred in a
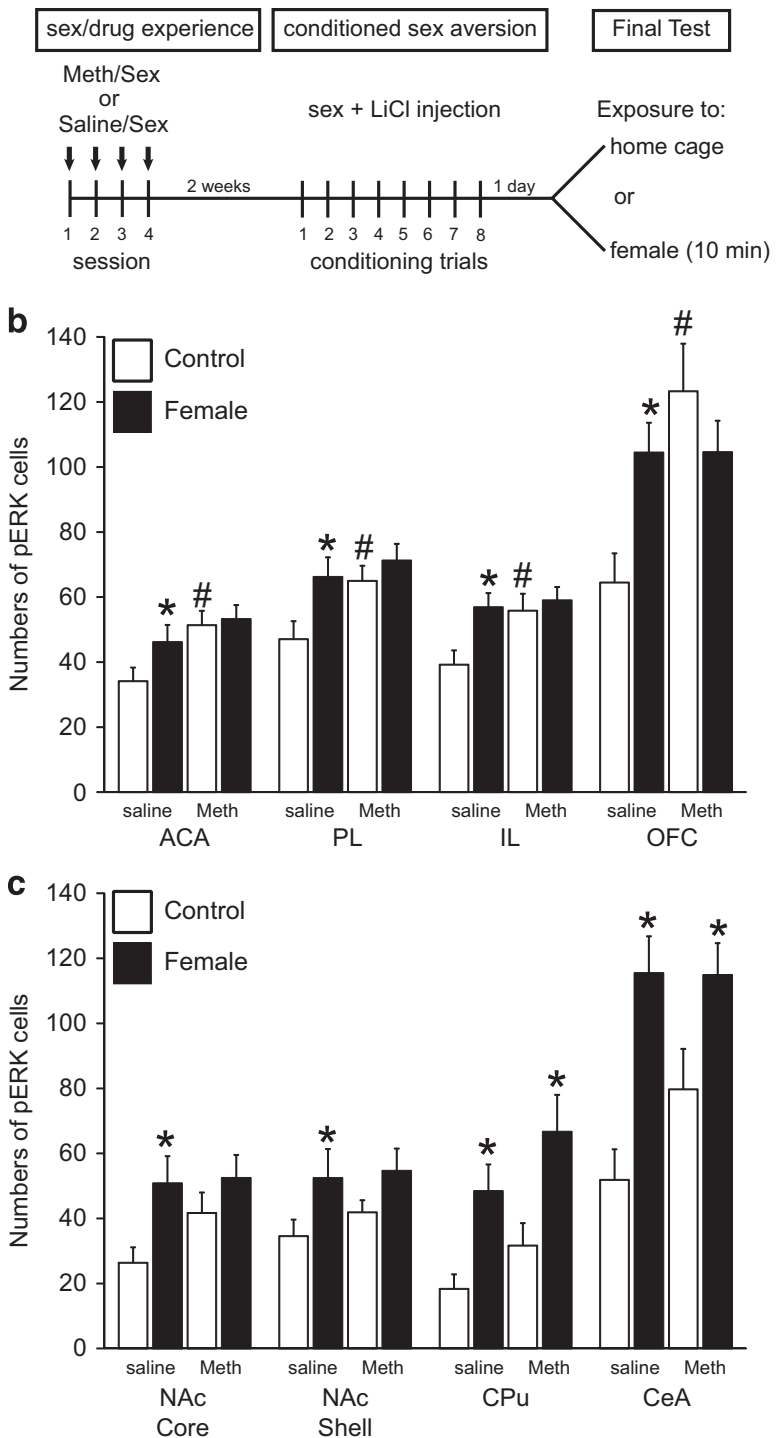

Figure 3 Effect of Meth/sex pretreatment on conditioned cue-induced PERK. (a) Experimental design and timeline of experiment 2, showing 4 days of Saline/sex or Meth/sex treatment, followed 2 weeks later by 8 conditioning trials of conditioned sex aversion (CSA), and the final exposure to home cage or female for phosphorylated MAP kinase ( $P E R K)$ analysis. $(b, c)$ Numbers of $p E R K$-immunoreactive neurons per $\mathrm{mm}^{2}$ (mean $\left.\pm \mathrm{SEM}\right)$ in males pretreated with Saline/sex (saline), or Meth/sex (Meth) and removed from home cage (control; white bars; $n=12$ saline and $n=10$ Meth) or exposed to the female (female; black bars; $n=14$ saline and $n=17$ Meth). (b) pERK in mPFC subregions and OFC, and (c) in NAc, CPU and CeA. ACA, anterior cingulate area; PL, prelimbic cortex; IL, infralimbic cortex; OFC, orbitofrontal cortex; NAc, nucleus accumbens; CPU, caudate putamen; VTA, ventral tegmental area; LA, lateral amygdala; BLA, basolateral amygdala; CeA, central amygdala. *Significant difference from home cage control group, " Significant difference from Saline/sex-pretreated home cage control group.

CaMKII neurons in the mPFC and OFC and may thus influence the efferent connections of these cortical areas.

First, it was demonstrated that exposure to cues associated with conditioned inhibition of sex behavior resulted in significant pERK activation in $\mathrm{mPFC}$ subregions, OFC, NAc, $\mathrm{CPu}$, and $\mathrm{CeA}$. This neural activation is distinct from that 

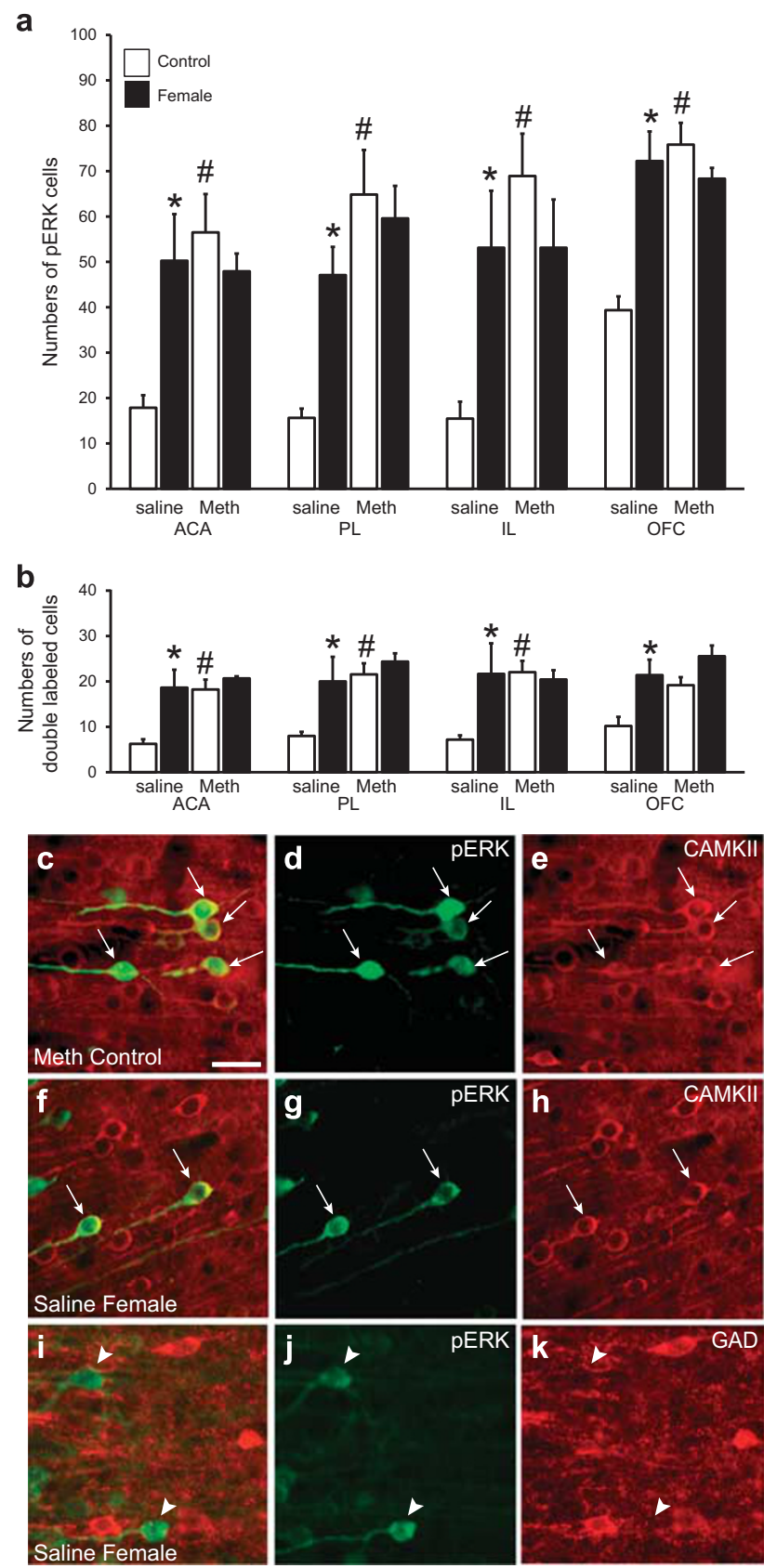

Figure 4 Phosphorylated MAP kinase (pERK) is localized in CaMKII neurons in medial prefrontal cortex (mPFC) and orbitofrontal cortex (OFC). (a) Numbers of pERK-immunofluorescent neurons per $\mathrm{mm}^{2}$ (mean \pm SEM) in $\mathrm{mPFC}$ subregions (ACA, PL, IL) and OFC in males (from experiment 2) pretreated with Saline/sex (saline), or Meth/sex (Meth) and removed from home cage (control; white bars; $n=4$ saline and $n=4$ Meth) or exposed to the female (female; black bars; $n=4$ saline and $n=3$ Meth). (b) Numbers of neurons co-labeled for pERK and CaMKII per $\mathrm{mm}^{2}$ (mean \pm SEM). ( $c-k)$ Representative images illustrating the co-localization (c, f; arrows indicate dual labeled neurons) of pERK (green; d,g,j) and CaMKII (red; e,h) and the lack of co-localization (i; arrow heads indicate pERK singlelabeled neurons) of pERK (green; j) and GAD (red; k) in PL of representative Meth+Sex Home Cage control (c-e), or Saline/sex exposed to female $(\mathrm{f}-\mathrm{k})$ males. Scale bar indicates $50 \mathrm{\mu m}$.

induced by exposure to a receptive female or mating behavior (Veening and Coolen, 2014) and thus appears to be involved in conditioned inhibition rather than initiation of sex behavior. The current findings expand previous reports showing pERK in NAc and $\mathrm{mPFC}$ during expression and acquisition of conditioned taste aversion, respectively (Marotta et al, 2014). The current findings suggest that $\mathrm{mPFC}$ is involved in the expression of CSA, having an extended role in regulation of mating inhibition beyond a role in learning (Calu et al, 2013; Riga et al, 2014; Rosen et al, 2015). Indeed, lesions of the mPFC result in expression of maladaptive sex behavior, without disruption of aversive learning or the expression of sexual behavior and reward (Davis et al, 2010).

Moreover, the current findings show that Meth and sex experience prevented the induction of neural activation by CSA-related cues in the MPFC and OFC, further demonstrating the potential involvement of these frontal cortices in the effects of Meth and sex experience on maladaptive sex behavior. This is consistent with roles for OFC and $\mathrm{mPFC}$ in cue-outcome prediction and expectancy (OFC) (Schoenbaum and Shaham, 2008), and decision making and associative learning (mPFC) (Riga et al, 2014).

Imaging studies using functional fMRI in humans with compulsive sexual behavior have shown dorsal anterior cingulate area activation by exposure to sexually explicit cues, suggesting a disruption of PFC functionality (Voon et al, 2014). This disruption is associated with enhanced attentional bias towards sexually explicit cues (Mechelmans et al, 2014), consistent with a role for PFC in attentional shifting (Morris et al, 2016). Moreover, this disruption mostly affected frontal-striatal circuitries (Mechelmans et al, 2014; Morris et al, 2016). In the current study, Meth and sex experience also significantly attenuated pERK induction by conditioned cues in the NAc, an area critical for goaldirected behavior (NAc) (Cruz et al, 2014; Penner and Mizumori, 2012), but not in the $\mathrm{CPu}$, an area involved in acquisition and storage of motor habits (Atallah et al, 2007; Balleine et al, 2009). Therefore, in rat, maladaptive sex behavior associated with Meth/sex experience appears to affect a frontal-striatal substrate that includes mPFC, OFC, and NAc. Finally, CeA expressed pERK induced by aversive conditioned cues, but was not altered by Meth/sex experience, consistent with the finding that conditioned aversion learning per se is not disrupted by Meth/sex experience (Frohmader et al, 2011). Instead, pERK in the CeA may be reflective of conditioned stress responses (Phelps and LeDoux, 2005).

A third main finding of the present study was the chronically increased baseline pERK expression after Meth/ sex pre-exposure. This effect was dependent on concurrent exposure to Meth/sex and associated with maladaptive sex behavior even in animals that were not pretreated with Meth. It is currently unclear what the exact consequences are of the increased baseline ERK activity, but pERK was expressed predominantly in CaMKII-expressing neurons, thereby presumably influencing $\mathrm{mPFC}$ output. The $\mathrm{mPFC}$ in turn suppresses dopamine transmission in the NAc thereby modulating motivated behaviors (Deisseroth, 2014). Hence, dysfunction of the mPFC output may contribute to altered dopamine function in the NAc, disrupted expression of learned responses, and increasing maladaptive goal-directed behaviors. Finally, the causes of the chronic elevation in ERK activation are unknown. Drugs alter neurotransmitter signaling in the mPFC, including serotonin (Muller et al, 2007), dopamine (Wise, 1998), and glutamate (Kalivas, 2009), as 
well as growth factors like BDNF (Grimm et al, 2003), but the role of these neurotransmitters in the effects of concurrent Meth and sex on maladaptive sex behavior are unknown. Finally, increased baseline pERK expression may have occurred in the same mPFC and OFC neurons as those responding to aversive cues, thereby contributing to the attenuation of further activation of these neurons and subsequent disruption of behavioral inhibition.

Together, these findings demonstrate a discrete neural substrate involved in inhibition of sexual behavior, which is functionally altered in animals with maladaptive sex behavior triggered by concurrent Meth and sex experience. Chronic alterations of ERK activity appear to be critical to these effects. Previous studies have shown that MEK inhibitors can prevent cue-induced drug seeking and increase extinction learning ( $\mathrm{Lu}$ et al, 2006; Papale et al, 2016; Valjent et al, 2006a). Therefore, MEK inhibitors may be promising avenues for treatment of drug-induced compulsive sex behavior.

\section{FUNDING AND DISCLOSURE}

This research was supported by a grant from the Intramural Research Support Program at the University of Mississippi Medical Center. The authors declare no conflict of interest.

\section{ACKNOWLEDGMENTS}

We thank Mr S. Rollins and Ms T. Smith for their technical support.

\section{REFERENCES}

Agmo A (2002). Copulation-contingent aversive conditioning and sexual incentive motivation in male rats: evidence for a two-stage process of sexual behavior. Physiol Behav 77: 425-435.

Atallah HE, Lopez-Paniagua D, Rudy JW, O’Reilly RC (2007). Separate neural substrates for skill learning and performance in the ventral and dorsal striatum. Nat Neurosci 10: 126-131.

Balleine BW, Liljeholm M, Ostlund SB (2009). The integrative function of the basal ganglia in instrumental conditioning. Behav Brain Res 199: 43-52.

Berman DE, Hazvi S, Rosenblum K, Seger R, Dudai Y (1998). Specific and differential activation of mitogen-activated protein kinase cascades by unfamiliar taste in the insular cortex of the behaving rat. J Neurosci 18: 10037-10044.

Calu DJ, Kawa AB, Marchant NJ, Navarre BM, Henderson MJ, Chen B et al (2013). Optogenetic inhibition of dorsal medial prefrontal cortex attenuates stress-induced reinstatement of palatable food seeking in female rats. J Neurosci 33: 214-226.

Cartier JJ, Greenwell L, Prendergast ML (2008). The persistence of HIV risk behaviors among methamphetamine-using offenders. J Psychoactive Drugs 40: 437-446.

Cruz FC, Babin KR, Leao RM, Goldart EM, Bossert JM, Shaham Y et al (2014). Role of nucleus accumbens shell neuronal ensembles in context-induced reinstatement of cocaine-seeking. J Neurosci 34: 7437-7446.

Davis JF, Loos M, Di Sebastiano AR, Brown JL, Lehman MN, Coolen LM (2010). Lesions of the medial prefrontal cortex cause maladaptive sexual behavior in male rats. Biol Psychiatry 67: 1199-1204.

Deisseroth K (2014). Circuit dynamics of adaptive and maladaptive behaviour. Nature 505: 309-317.
Fanous S, Goldart EM, Theberge FR, Bossert JM, Shaham Y, Hope BT (2012). Role of orbitofrontal cortex neuronal ensembles in the expression of incubation of heroin craving. J Neurosci 32: $11600-11609$.

Frohmader KS, Bateman KL, Lehman MN, Coolen LM (2010a). Effects of methamphetamine on sexual performance and compulsive sex behavior in male rats. Psychopharmacology 212: 93-104.

Frohmader KS, Lehman MN, Laviolette SR, Coolen LM (2011). Concurrent exposure to methamphetamine and sexual behavior enhances subsequent drug reward and causes compulsive sexual behavior in male rats. J Neurosci 31: 16473-16482.

Frohmader KS, Pitchers KK, Balfour ME, Coolen LM (2010b). Mixing pleasures: review of the effects of drugs on sex behavior in humans and animal models. Horm Behav 58: 149-162.

Frohmader KS, Wiskerke J, Wise RA, Lehman MN, Coolen LM (2010c). Methamphetamine acts on subpopulations of neurons regulating sexual behavior in male rats. Neuroscience 166: 771-784.

Giorgi O, Corda MG, Sabariego M, Giugliano V, Piludu MA, Rosas M et al (2015). Differential effects of cocaine on extracellular signalregulated kinase phosphorylation in nuclei of the extended amygdala and prefrontal cortex of psychogenetically selected Roman high- and low-avoidance rats. J Neurosci Res 93: 714-721.

Green AI, Halkitis PN (2006). Crystal methamphetamine and sexual sociality in an urban gay subculture: an elective affinity. Cult Health Sex 8: 317-333.

Grimm JW, Lu L, Hayashi T, Hope BT, Su TP, Shaham Y (2003). Time-dependent increases in brain-derived neurotrophic factor protein levels within the mesolimbic dopamine system after withdrawal from cocaine: implications for incubation of cocaine craving. J Neurosci 23: 742-747.

Harlow E, Lane D (1988). Antibodies, A Laboratory Manual. Cold Spring Harbor Laboratory Press: Cold Spring Harbor, NY, USA.

Johnson MW, Bruner NR (2012). The sexual discounting task: HIV risk behavior and the discounting of delayed sexual rewards in cocaine dependence. Drug Alcohol Depend 123: 15-21.

Johnson MW, Johnson PS, Herrmann ES, Sweeney MM (2015). Delay and probability discounting of sexual and monetary outcomes in individuals with cocaine use disorders and matched controls. PLoS One 10: e0128641.

Kalivas PW (2009). The glutamate homeostasis hypothesis of addiction. Nat Rev Neurosci 10: 561-572.

Kraus SW, Voon V, Potenza MN (2016). Neurobiology of compulsive sexual behavior: emerging science. Neuropsychopharmacology 41: 385-386.

Longoni R, Spina L, Vinci S, Acquas E (2011). The MEK inhibitor SL327 blocks acquisition but not expression of lithium-induced conditioned place aversion: a behavioral and immunohistochemical study. Psychopharmacology 216: 63-73.

Lu L, Koya E, Zhai H, Hope BT, Shaham Y (2006). Role of ERK in cocaine addiction. Trends Neurosci 29: 695-703.

Lyons T, Chandra G, Goldstein J, Ostrow DG (2010). Breaking the bond between stimulant use and risky sex: a qualitative study. Subst Abus 31: 224-230.

Marotta R, Fenu S, Scheggi S, Vinci S, Rosas M, Falqui A et al (2014). Acquisition and expression of conditioned taste aversion differentially affects extracellular signal regulated kinase and glutamate receptor phosphorylation in rat prefrontal cortex and nucleus accumbens. Front Behav Neurosci 8: 153.

Mastroleo NR, Operario D, Barnett NP, Colby SM, Kahler CW, Monti PM (2015). Prevalence of heavy drinking and risky sexual behaviors in adult emergency department patients. Alcohol Clin Exp Res 39: 1997-2002.

Mausbach BT, Semple SJ, Strathdee SA, Patterson TL (2009). Predictors of safer sex intentions and protected sex among heterosexual HIV-negative methamphetamine users: an expanded model of the theory of planned behavior. AIDS Care 21: 17-24. 
Mechelmans DJ, Irvine M, Banca P, Porter L, Mitchell S, Mole TB et al (2014). Enhanced attentional bias towards sexually explicit cues in individuals with and without compulsive sexual behaviours. PLoS One 9: e105476.

Mitchell SG, Kelly SM, Brown BS, O’Grady KE, Schwartz RP (2012). HIV sex-risk behaviors among in- versus out-of-treatment heroin-addicted adults. Am J Drug Alcohol Abuse 38: 328-333.

Morris LS, Kundu P, Dowell N, Mechelmans DJ, Favre P, Irvine MA et al (2016). Fronto-striatal organization: defining functional and microstructural substrates of behavioural flexibility. Cortex 74: 118-133.

Muller CP, Carey RJ, Huston JP, De Souza Silva MA (2007). Serotonin and psychostimulant addiction: focus on 5-HT1Areceptors. Prog Neurobiol 81: 133-178.

Papale A, Morella IM, Indrigo MT, Eugene Bernardi R, Marrone L, Marchisella F et al (2016). Impairment of cocaine-mediated behaviours in mice by clinically relevant Ras-ERK inhibitors. Elife 5: e17111.

Penner MR, Mizumori SJ (2012). Neural systems analysis of decision making during goal-directed navigation. Prog Neurobiol 96: 96-135.

Peters RH (1983). Learned aversions to copulatory behaviors in male rats. Behav Neurosci 97: 140-145.

Phelps EA, LeDoux JE (2005). Contributions of the amygdala to emotion processing: from animal models to human behavior. Neuron 48: 175-187.

Pitchers KK, Coppens CM, Beloate LN, Fuller J, Van S, Frohmader KS et al (2014). Endogenous opioid-induced neuroplasticity of dopaminergic neurons in the ventral tegmental area influences natural and opiate reward. J Neurosci 34: 8825-8836.

Ribeiro MJ, Schofield MG, Kemenes I, O'Shea M, Kemenes G, Benjamin PR (2005). Activation of MAPK is necessary for longterm memory consolidation following food-reward conditioning. Learn Mem 12: 538-545.

Riga D, Matos MR, Glas A, Smit AB, Spijker S, Van den Oever MC (2014). Optogenetic dissection of medial prefrontal cortex circuitry. Front Syst Neurosci 8: 230.
Rosen LG, Sun N, Rushlow W, Laviolette SR (2015). Molecular and neuronal plasticity mechanisms in the amygdala-prefrontal cortical circuit: implications for opiate addiction memory formation. Front Neurosci 9: 399.

Schafe GE, Atkins CM, Swank MW, Bauer EP, Sweatt JD, LeDoux JE (2000). Activation of ERK/MAP kinase in the amygdala is required for memory consolidation of pavlovian fear conditioning. J Neurosci 20: 8177-8187.

Schoenbaum G, Shaham Y (2008). The role of orbitofrontal cortex in drug addiction: a review of preclinical studies. Biol Psychiatry 63: 256-262.

Semple SJ, Patterson TL, Grant I (2004). The context of sexual risk behavior among heterosexual methamphetamine users. Addict Behav 29: 807-810.

Takamura H, Ichisaka S, Watanabe K, Toigawa M, Hata Y (2008). Effects of anesthesia on immunohistochemical detection of phosphorylated extracellular signal-regulated kinase in cerebral cortex. J Neurosci Methods 170: 300-304.

Valjent E, Corbille AG, Bertran-Gonzalez J, Herve D, Girault JA (2006a). Inhibition of ERK pathway or protein synthesis during reexposure to drugs of abuse erases previously learned place preference. Proc Natl Acad Sci USA 103: 2932-2937.

Valjent E, Corvol JC, Trzaskos JM, Girault JA, Herve D (2006b). Role of the ERK pathway in psychostimulant-induced locomotor sensitization. BMC Neurosci 7: 20.

Veening JG, Coolen LM (2014). Neural mechanisms of sexual behavior in the male rat: emphasis on ejaculation-related circuits. Pharmacol Biochem Behav 121: 170-183.

Voon V, Mole TB, Banca P, Porter L, Morris L, Mitchell S et al (2014). Neural correlates of sexual cue reactivity in individuals with and without compulsive sexual behaviours. PLoS One 9: e102419.

Wise RA (1998). Drug-activation of brain reward pathways. Drug Alcohol Depend 51: 13-22.

Zapata LB, Hillis SD, Marchbanks PA, Curtis KM, Lowry R (2008). Methamphetamine use is independently associated with recent risky sexual behaviors and adolescent pregnancy. J Sch Health 78: 641-648.

Supplementary Information accompanies the paper on the Neuropsychopharmacology website (http://www.nature.com/npp) 